\title{
Operational modal testing and analysis: an effective tool for the assessment of masonry towers
}

\author{
N. Gallino, C. Gentile \& A. Saisi \\ Politecnico di Milano, DIS - Department of Structural Engineering, Italy
}

\begin{abstract}
This paper presents the results of a recent research on the dynamic behaviour of an historic bell-tower, built in stonework masonry. The first part of the investigation involved: (a) an accurate survey of the tower geometry and of the crack pattern; (b) ambient vibration testing under wind, micro-tremors and swinging of bells; (c) modal identification; (d) FE modelling and model tuning.

Since the amplitude of the excitation seems to affect the modal behaviour of the structure, a permanent dynamic monitoring system was recently installed inside the tower in order to investigate the changes in the modal parameters and the possible correlation with damage.
\end{abstract}

Keywords: ambient vibration testing, historic structures, modal parameters, monitoring.

\section{Introduction}

In recent decades, the preservation strategies of Historic Architectural Heritage more and more frequently involved the diagnosis of the historic buildings based on non destructive testing (see e.g. Binda [1]). Peculiarly, the investigations on history, geometry, materials and damage provide a first diagnosis, to be further refined by using Finite Element (FE) analyses. The FE model of an historic structure, even when based on an accurate field survey, always involves simplifying assumptions and several uncertainties in the material, the geometric properties and boundary conditions. Within this context, one possible key role of ambient vibration testing (AVT) and operational modal analysis (OMA) is to provide an effective and accurate validation of the model prior to its use in numerical analysis. 
AVT and OMA are especially suitable for historic structures, for several reasons: (a) the easy and non destructive way of testing, performed by measuring only the structural response under ambient excitation; (b) the sustainability of testing, which does not interfere with the normal use of the structure; (c) the multiple-input nature and the wide-band frequency content of the excitation, ensuring that the response includes the contribution of a significant number of modes; (d) the large number of highly sensitive $(10 \mathrm{~V} / \mathrm{g})$ piezoelectric or forcebalanced accelerometers available on the market and relatively inexpensive; (e) the large number of output-only modal identification techniques available in the literature, ranging from the simple Peak Picking technique (Bendat [2]) to the more advanced Frequency Domain Decomposition (Brincker [3]) and Stochastic Subspace Identification (Van Overschee [4]). Another possible application of OMA in the field of historic structures is continuous or discrete monitoring in order to perform dynamics-based damage assessment (Ramos [5]).

This paper presents the analytical and experimental dynamic analysis of an historic masonry bell-tower, about $37.0 \mathrm{~m}$ high and placed in the small town of Arcisate (about $80 \mathrm{~km}$ from Milan, Italy). Two dynamic tests in operational conditions were carried out in 2007 and 2008, respectively. Each experimental program of field surveys included two series of ambient vibration tests: in the first series, the excitation was only provided by wind and micro-tremors, while in the second series "random" swinging of bells provided the excitation. Five modes of vibration were clearly identified in the frequency range $0-6 \mathrm{~Hz}$ by using different OMA techniques and the identified modal parameters seem to exhibit dependence on the amplitude of excitation. Subsequently, a FE model of the tower was developed for safety evaluation and a simple permanent monitoring system (using three highly sensitive piezoelectric accelerometers) was placed inside the tower in order to investigate the variation of modal parameters and the possible correlation with damage.

\section{The "Collegiata of San Vittore" bell tower in Arcisate}

The investigated bell tower (Fig. 1), about $37.0 \mathrm{~m}$ high, is built in stonework masonry and connected, on the East side and partly on the South side, to the Church of San Vittore (XI century) in Arcisate (Varese). The first historic document on the tower dates back to XVI century, even though it was built on a previous roman building and modified along the centuries.

As shown in the pictures of Fig. 1, the tower is characterized by irregular stonework. In the upper part, six orders of floors are present, with five of them being defined by masonry offsets at the corners and by corresponding sequences of small hanging arches marking the floor levels; the last two orders were probably added in the 18th century to host the bell trusses.

The masonry texture appears often highly disordered with the local prevalence of continuous or little staggered vertical joints; in addition, due to erosion of the mortar joints, it is difficult to distinguish between insufficient stone interlocking and vertical cracks. The crack pattern (Fig. 1) has been accurately surveyed also by using an aerial platform, which allowed to closely inspecting the external wall surface and detecting the flaw paths. Along all sides, the tower exhibits long vertical cracks, most of them cutting the entire wall 
thickness and passing through the keystones of the arch window openings. These cracks are especially detected between the second / third order of the tower and the base of the belfry and show a maximum aperture corresponding to the upper end. Many superficial cracks are also diffused, particularly on the North and West fronts (Fig. 1), which are not adjacent to the church.

The masonry has been also characterized through sonic tests and the results of sonic velocity generally indicate a relatively compact masonry, of fairly good execution.

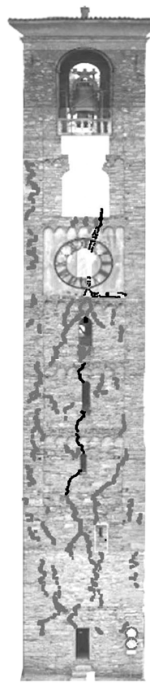

North Side

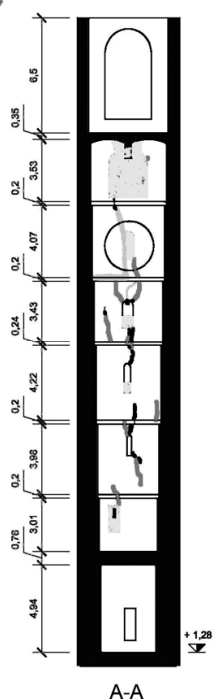

A-A

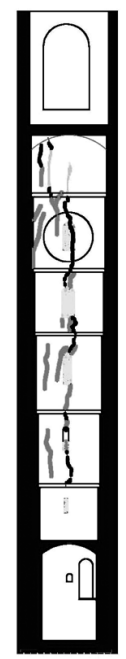

B-B

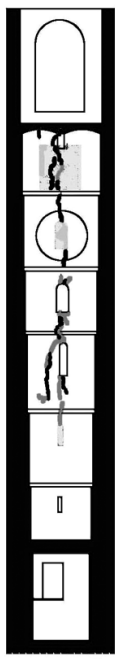

C-C

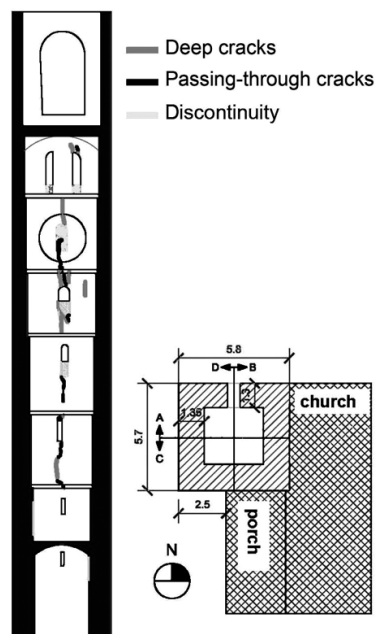

D-D

Figure 1: Crack pattern on the north side of the tower and on sections (dimensions in $\mathrm{m}$ ).

\section{Ambient vibration tests and modal identification}

\subsection{Testing procedures}

Two ambient vibration tests were conducted on the tower, on June 2007 and June 2008, respectively. In both tests, a 16-channel data acquisition system with 15 uniaxial WR 731A piezoelectric accelerometers (Fig. 2(b)) were used; the response of the tower was measured in 15 selected points, according to the sensor layout shown in Fig. 2(a). For each test, two different series of ambient vibration data were recorded: (i) in the first series, the ambient excitation was only provided by the wind and the micro-tremors; (ii) in the second series, the excitation was provided by the swinging of bells.

In the following, these two different series of ambient vibration data will be referenced to as AV1 (micro-tremors and wind) and AV2 (swinging of bells). In both series, the well-known condition (see e.g. Cantieni [6]) about the length of the time windows acquired (that should be at least 1000 times the period of the 
structure's fundamental mode) was largely satisfied. The sample rate was $200 \mathrm{~Hz}$ to provide good waveform definition.

An example of the acceleration time-histories recorded in June 2007 test at channel 12 (Fig 2(a)) is given in Figs. 3(a) and (b). It should be noticed that the bell swinging produced a significant increase of the vibration level associated with micro-tremors and other ambient excitations, as it is shown in Figs. 3(a) and (b); it can be observed that the maximum amplitude of acceleration responses is increased of 10-20 times by the bell swinging. Similar results have been obtained in the test performed on June 2008.

\subsection{Modal identification techniques}

The extraction of modal parameters from ambient vibration data was carried out by using two different output-only techniques: the Frequency Domain Decomposition (FDD, Brincker [3]) in the frequency domain and the data driven Stochastic Subspace Identification (SSI, Van Overschee [4]) in the time domain; these techniques are available in the commercial program ARTeMIS [7].

The FDD technique involves the following steps: (a) estimate of the spectral matrix $\boldsymbol{G}(f)$ (i.e. the matrix of cross-spectral densities); (b) Singular Value Decomposition (SVD) of $\boldsymbol{G}(f)$ at each frequency; (c) inspection of the curves representing the SVs to identify the resonant frequencies and estimate the corresponding mode shape using the information contained in the singular vectors of the SVD.

Since the first (and largest) singular value at each frequency represents the strength of the dominating vibration mode at that frequency, plotting the first

(a)

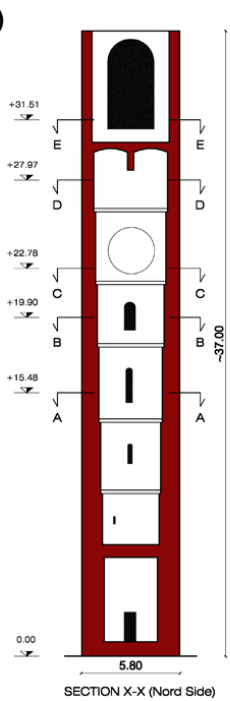

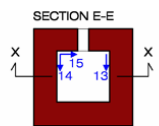

SECTION D-D

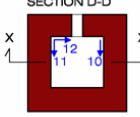

SECTIONC-C
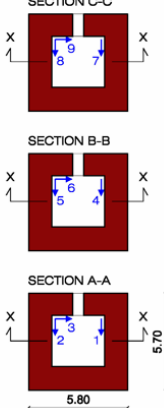

(b)

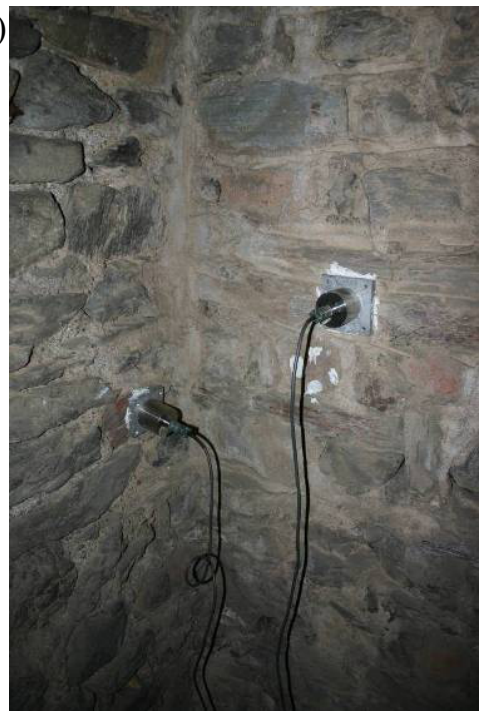

Figure 2: $\quad$ (a) Sensor layout adopted in AVT of the tower (dimensions in $\mathrm{m}$ );

(b) Example of WR-731A accelerometers attached to the tower. 
(a)

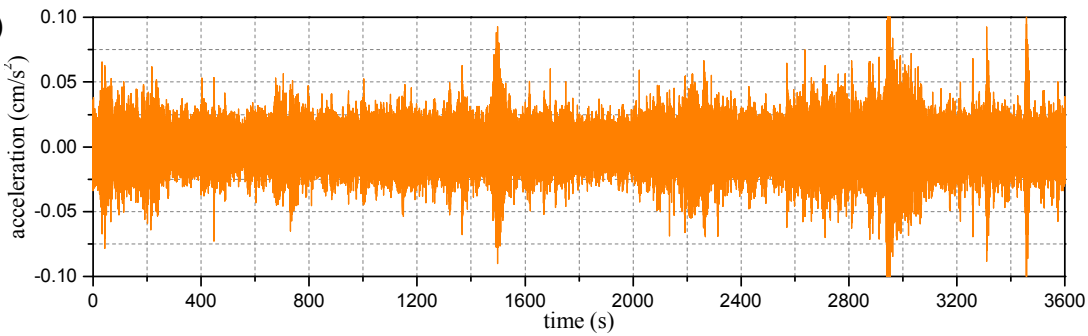

(b)

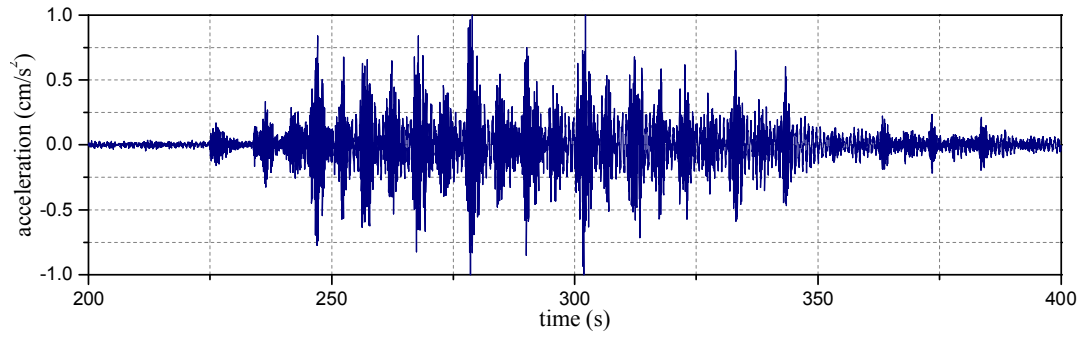

Figure 3: Acceleration induced at Ch. 12 by: (a) micro-tremors and wind; (b) swinging of bells.

singular value yields the resonant frequencies as local maxima, as it is shown in Figs. 4(a) and 6 for the investigated tower. It is worth noting that the FDD technique is able to detect closely spaced modes; in such instances, more than a singular value will reach a maximum in the neighbourhood of a given frequency and every singular vectors corresponding to a non-zero singular value is a mode shape estimate.

The SSI method works in the time domain and is based on the discrete-time state-space form of the dynamics of a linear-time-invariant system under unknown excitation:

$$
\begin{gathered}
\boldsymbol{x}_{k+1}=\boldsymbol{A} \boldsymbol{x}_{k}+\boldsymbol{w}_{k} \\
\boldsymbol{y}_{k}=\boldsymbol{C} \boldsymbol{x}_{k}+\boldsymbol{v}_{k}
\end{gathered}
$$

where $\boldsymbol{x}_{k}$ is the discrete-time state vector (containing displacements and velocities describing the state of the system at time instant $t_{k}=k \Delta t$ ), $\boldsymbol{w}_{k}$ is the process noise, $\boldsymbol{y}_{k}$ is the output vector, $\boldsymbol{v}_{k}$ is the measurement noise, $\boldsymbol{A}$ is the discrete state matrix (dependent on the mass, stiffness and damping properties of the structure) and $\boldsymbol{C}$ is the discrete output matrix (which maps the state vector into the measured output). Eq. (1) is generally called the state equation while Eq. (2) is called the observation/output equation.

It can be shown (Peeters [8]) that the modal parameters (natural frequencies, mode shapes and damping ratios) of a structure under white-noise excitation can be identified by relying only on the measured output responses $\boldsymbol{y}_{k}$. Once state space matrices are identified of different order $N, N / 2$ modal parameters are extracted from a model of order $N$. If similar modal parameters are obtained with increasing model order, a physical eigenmode is identified. In the present application, stochastic state space models are identified of different order $N$, ranging from 2 to 80 in steps of 2 . 


\subsection{Mode shapes correlation}

In order to compare the mode shapes identified using different methods and different test data, the Modal Assurance Criterion (MAC, Allemang [9]) was computed. The MAC is probably the most commonly used procedure to correlate two sets of mode shape vectors and is defined as:

$$
\operatorname{MAC}\left(\boldsymbol{\phi}_{A, k}, \boldsymbol{\phi}_{B, j}\right)=\frac{\left(\boldsymbol{\phi}_{A, k}^{T} \boldsymbol{\phi}_{B, j}\right)^{2}}{\left(\boldsymbol{\phi}_{A, k}^{T} \boldsymbol{\phi}_{A, k}\right)\left(\boldsymbol{\phi}_{B, j}^{T} \boldsymbol{\phi}_{B, j}\right)}
$$

where $\phi_{A, k}$ is the $k$-th mode of data set A and $\phi_{B, j}$ the $j$-th mode of the data set B. The MAC value is a coefficient analogous to the correlation coefficient in statistics and ranges from 0 to 1 ; a value of 1 implies perfect correlation of the two mode shape vectors (one vector is a multiple of the other) while a value close to 0 indicates uncorrelated (orthogonal) vectors. In general, a MAC value greater than $0.80-0.85$ is considered a good match while a MAC value less than 0.40 is considered a poor match.

(a)

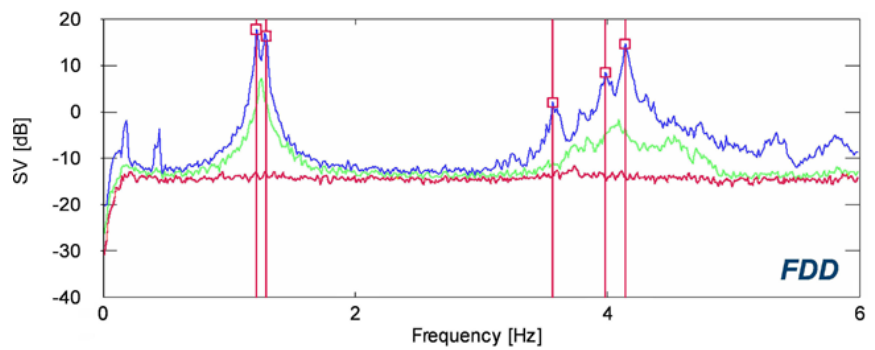

(b)

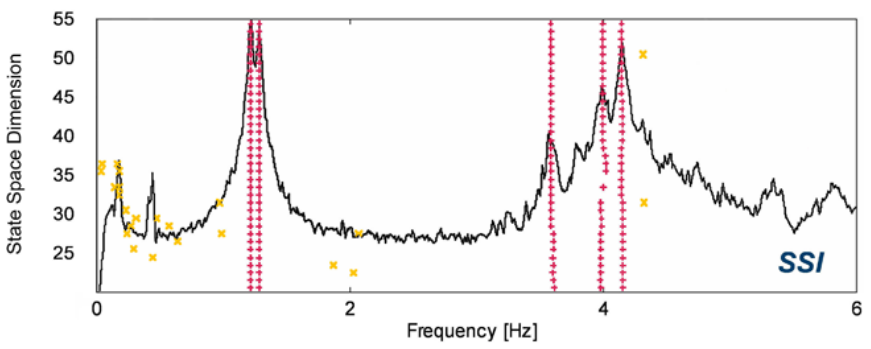

Figure 4: Data series AV1, June 2007: (a) singular values of the spectral matrix and selected modes (FDD technique); (b) stabilization diagram (SSI technique).

\section{Dynamic characteristics of the tower}

The application of both FDD and SSI techniques to AV1 data recorded in June 2007 leads to the identification of 5 vibration modes in the frequency range of 0 $6 \mathrm{~Hz}$. The results of OMA in terms of natural frequencies can be summarized through the plots of Figs. 4(a) and (b); the figures show the lower SVs of the spectral matrix and the stabilization diagrams obtained by applying the FDD and 
the SSI technique, respectively. The inspection of Fig. 4(a) clearly highlight that the FDD technique provides a clear indication of the tower modes through welldefined local maxima in the $1^{\text {st }} \mathrm{SV}$; similarly, Fig. 4(b) shows that the alignments of the stable poles in the stabilization diagram of the SSI method provides a clear indication of these modes, as well. Furthermore, Figs. 4(a) and (b) clearly show the correspondence of the natural frequency estimates between the two techniques, with the resonant peaks of Fig. 4(a) being placed practically at the same frequencies of the alignments of stable poles of Fig. 4(b).

As it had to be expected, the identified modes can be classified as bending and torsion. Fig. 5 shows the identified mode shapes (AV1, June 2007, FDD technique): dominant bending (B) modes were identified at $1.21\left(\mathrm{~B}_{1}\right), 1.29\left(\mathrm{~B}_{2}\right)$, $3.98\left(\mathrm{~B}_{3}\right)$ and $4.14 \mathrm{~Hz}\left(\mathrm{~B}_{4}\right)$ while only one torsion mode $\left(\mathrm{T}_{1}\right)$ was identified at $3.56 \mathrm{~Hz}$. It is observed that the dominant bending modes of the tower involve flexure practically along the diagonals.

Since two datasets, acquired under different level of ambient excitation, are available, it is very interesting to compare the estimates of the modal parameters identified from the two different datasets collected.

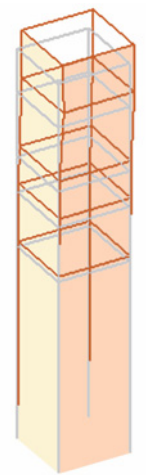

$f=1.211 \mathrm{~Hz}$

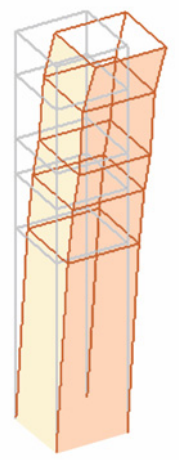

$f=1.289 \mathrm{~Hz}$

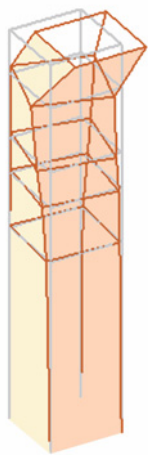

$f=3.564 \mathrm{~Hz}$

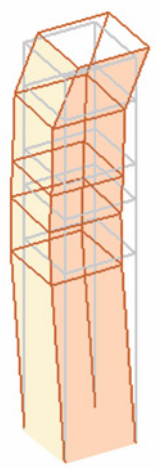

$f=3.984 \mathrm{~Hz}$

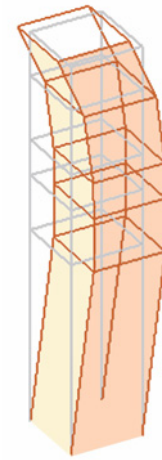

$f=4.141 \mathrm{~Hz}$

Figure 5: Vibration modes identified from data series AV1, June 2007 (FDD).

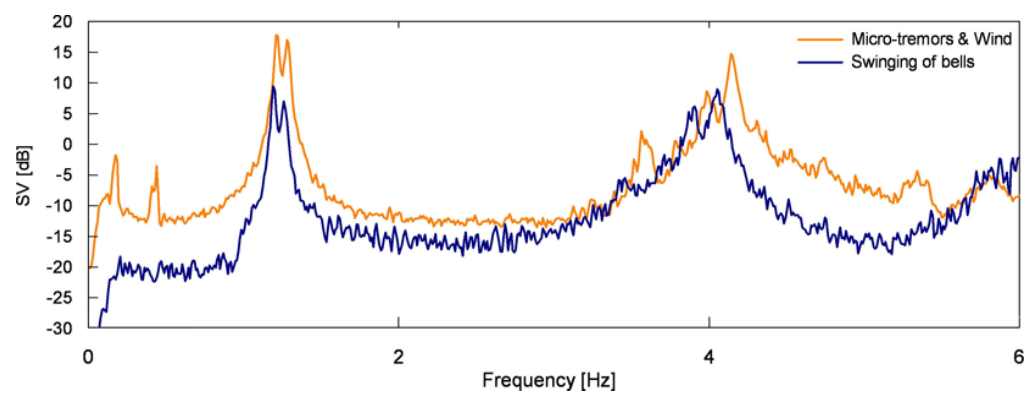

Figure 6: Comparison between the $1^{\text {st }} \mathrm{SV}$ of the spectral matrices obtained from data series AV1 and AV2 in June 2007. 
Fig. 6 shows the $1^{\text {st }} \mathrm{SV}$ lines of the spectral matrix obtained from data series AV1 and AV2 recorded in June 2007; the inspection of spectral plots reveals slight but clear decrease of the frequency of spectral peaks (resonant frequencies) associated to higher level of excitation that existed during the bell swinging (Table 1). Furthermore, significant differences are detected between the mode shapes identified from data series AV1 and AV2. Similar results were obtained in the test performed on June 2008, even if with less marked differences in the frequency decreasing. The superposition of the mode shapes identified in the two series of tests clearly highlights that the MAC in the 2007 test tends to decrease as the order of mode increases; for the two upper modes the MAC is between 0.87 and 0.80 . In the June 2008 test, the MAC seems more stable.

Hence, the dynamic characteristics of the tower are possibly dependent on the amplitude of excitation/response. In order to better understand and investigate this peculiar behaviour, a continuous dynamic monitoring system (including 3 highly sensitive accelerometers) was recently mounted on the tower (Fig. 7).

Table 1: $\quad$ Correlation between the modal parameters identified in 2007 and 2008.

\begin{tabular}{|c|c|c|c|c||c|c|c|c|}
\cline { 2 - 9 } \multicolumn{1}{c|}{} & \multicolumn{4}{c||}{ June 2007} & \multicolumn{4}{c|}{ June 2008 } \\
\hline Mode N. & $\begin{array}{c}f_{\mathrm{AV} 1} \\
(\mathrm{~Hz})\end{array}$ & $\begin{array}{c}f_{\mathrm{AV} 2} \\
(\mathrm{~Hz})\end{array}$ & $\begin{array}{c}\Delta f / f \\
(\%)\end{array}$ & $M A C$ & $\begin{array}{c}f_{\mathrm{AV} 1} \\
(\mathrm{~Hz})\end{array}$ & $\begin{array}{c}f_{\mathrm{AV} 2} \\
(\mathrm{~Hz})\end{array}$ & $\begin{array}{c}\Delta f / f \\
(\%)\end{array}$ & $M A C$ \\
\hline \hline 1 & 1.211 & 1.191 & -1.65 & 0.997 & 1.211 & 1.201 & -0.83 & 0.982 \\
\hline 2 & 1.289 & 1.260 & -2.25 & 0.986 & 1.270 & 1.260 & -0.79 & 0.955 \\
\hline 3 & 3.564 & - & - & - & 3.525 & 3.467 & -1.65 & 0.942 \\
\hline 4 & 3.984 & 3.877 & -2.69 & 0.870 & 3.984 & 3.906 & -1.96 & 0.936 \\
\hline 5 & 4.141 & 4.059 & -1.98 & 0.804 & 4.141 & 4.063 & -1.88 & 0.982 \\
\hline
\end{tabular}
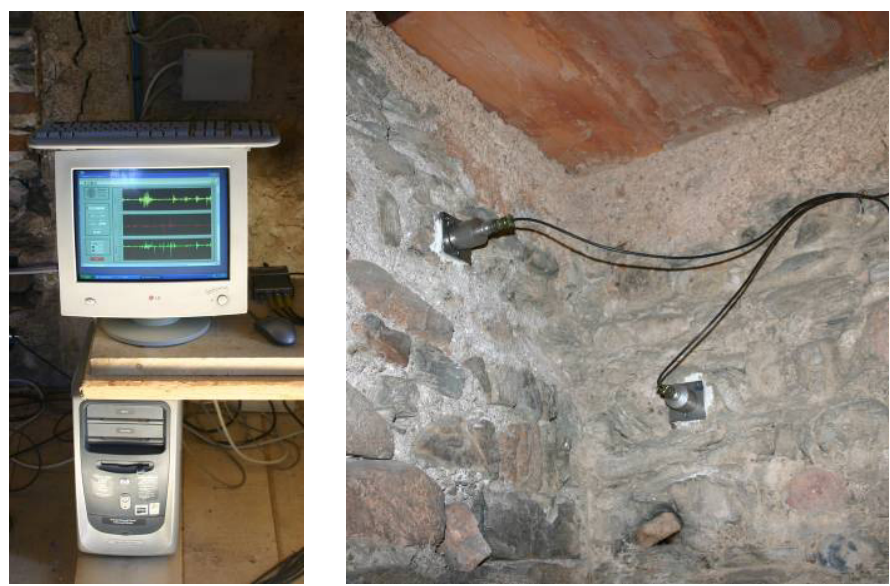

Figure 7: Details of the continuous monitoring acquisition system. 


\section{$5 \quad$ FE modelling and model tuning}

The experimental investigation was preceded by the development of a 3D finite element model (Fig. 8), based on the available geometric survey. The F.E. program SAP2000 was used to create the numerical model.

The tower was modelled by using 8 -node brick elements. A relatively large number of finite elements have been used in the model, so that a regular distribution of masses could be obtained and all the openings in the load-bearing walls could be reasonably represented. The model consists of 3481 solid elements with 17196 active degrees of freedom.

Since the geometry of the tower was accurately checked, the main uncertainties are related to the boundary conditions and the characteristics of the material. In order to reduce the number of uncertainties in the model calibration, the following assumptions were introduced: (a) the weight per unit volume of the masonry was assumed as $17.0 \mathrm{kN} / \mathrm{m}^{3}$; (b) the Poisson's ratio of the masonry was held constant and equal to 0.15 ; (c) since the soil-structure interaction is hardly involved at the low level of ambient vibrations that existed during the tests, the tower footing was considered as fixed. In such instances, the main uncertainties are related to:

1. the average elastic characteristics of stone masonry;

2. the connection between the tower and the neighbouring building (Fig. 1).

A preliminary dynamic analysis was performed to check the similarity between experimental and theoretical modal parameters. In this analysis, the Young's modulus of stone masonry was assumed equal to $3.00 \mathrm{GPa}$. It is worth noting that the assumed value was suggested either by engineering judgement or by the results of sonic tests because exhaustive tests to evaluate the mechanical characteristics of the stone masonry are not yet available. The correlation between the dynamic characteristics of the FEM models and the experimental results is evaluated via the average frequency discrepancy $J$ (see Fig. 9) and the maximum absolute frequency discrepancy $D_{F, \max }$ (see Fig. 9).

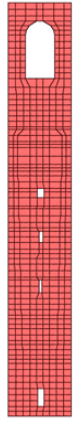

NORTH

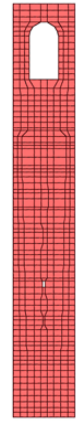

EAST

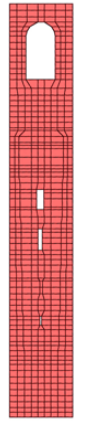

SOUTH

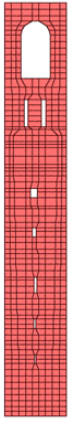

WEST

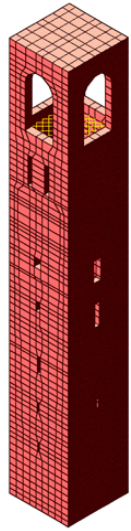

Figure 8: Finite element model of the tower. 
The comparison between theoretical and experimental modal parameters shows highly imperfect correlation, since the average frequency discrepancy ranges up to about $21.57 \%$ with the maximum value of $56.26 \%$. Beyond that, FEM1 does not accurately represent the structural behaviour of the tower since:

a) the model is much stiffer than the tower (with all the natural frequencies of

FEM1 model significantly exceeding the experimental ones);

b) the torsion mode $T_{1}$ of the model does not follow the experimental sequence, where the torsion mode is placed between two couples of bending modes (Fig. 5); on the contrary, the torsion mode follows two couples of bending modes in FEM1 model;

c) also the mode shapes of bending modes exhibit major differences with the experimental results. Specifically, FEM1 bending modes involve motion along the main N-S and E-W directions while the identified tower modes involve bending along the diagonals (Fig. 5).

The poor quality of correlation clearly indicates that the assumptions on the isotropic behaviour of stone masonry and on the connection with the neighbouring building need to be revised. Hence, an orthotropic elastic behaviour (FEM2) was assumed for the stone masonry; the average characteristics of the material were $E=3.00 \mathrm{GPa}, G_{13}=\mathrm{G}_{23}=0.33 \mathrm{GPa}$. The introduction of orthotropic elasticity dramatically improved the correlation with the experimental results. Specifically:

a) the stiffness of the model significantly decreased, so that the average and maximum frequency discrepancies are reduced from $21.57 \%$ to $8.97 \%$ and from $56.26 \%$ to $18.26 \%$, respectively;

b) the torsion mode $T_{1}$ of the FEM2 model correctly follows the experimental sequence.

On the other hand, the flexural modes of FEM2 continue to exhibit bending along the main N-S and E-W directions, differently from the observed modes.

A third model FEM3 was subsequently developed, by accounting for the connection between the tower and the church through a series of linear (nodal) springs of constant $k$. After some manual tuning, the stiffness of springs $k=$ $4 \times 10^{4} \mathrm{kN} / \mathrm{m}$ was assumed.
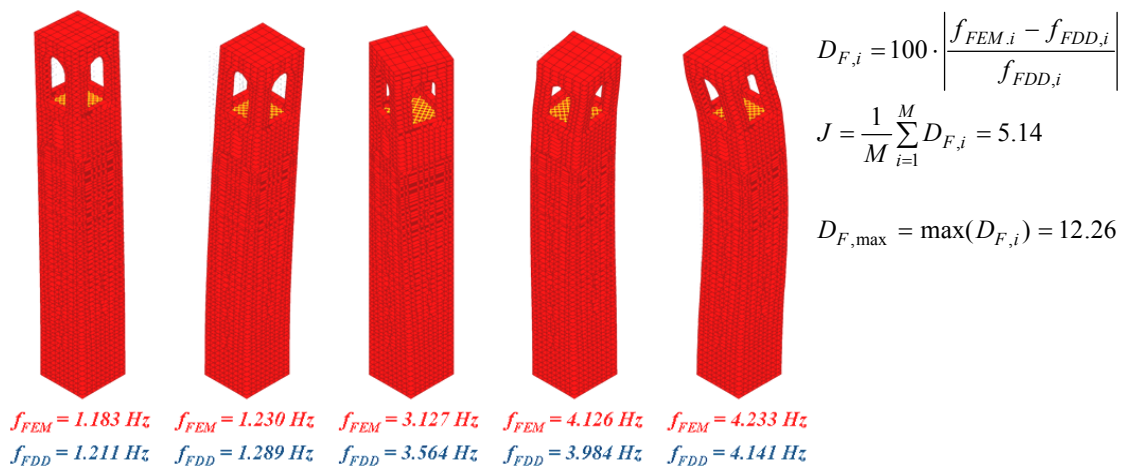

Figure 9: $\quad$ FEM3 (orthotropic material + springs): vibration modes. 
The correlation between the dynamic characteristics of FEM3 model and the experimental results is shown in Fig. 9; the figure also addresses the correlation between FEM3 and experimental modal characteristics, via the maximum absolute frequency discrepancy $D_{F, \max }$, and the average frequency discrepancy $J$. As it had to be expected from previous investigations (Gentile [10]), now the bending modes are fully consistent with the experimental results; in addition, further reduction of frequency discrepancies was attained, with $D_{F}$ being less than $5.35 \%$ for all bending modes. Of course, the value of $D_{F, \max }=12.26 \%$ (obtained for mode $\mathrm{T}_{1}$ ) remains quite high, as a consequence of the simplified distribution of the model elastic properties. However, the correlation between theoretical and experimental behaviour is satisfactory and provides a robust verification of the FEM3 model main assumptions, being a one-to-one correspondence between the mode shapes. Hence, the model seems suitable for a successive investigation, including further non-destructive tests on the materials, sensitivity analysis and application of system identification techniques.

\section{Conclusions}

Experimental and theoretical dynamic investigation of an historic masonry tower is described in the paper. The following conclusions can be drawn from the study:

1. Notwithstanding the very low level of ambient vibration level that may exist during the tests, AVT and OMA have proved to be effective tools for modal identification of masonry towers;

2. Within the frequency range $0-6 \mathrm{~Hz}, 4$ dominant bending and 1 torsion modes were clearly identified from ambient vibration data and very good agreement was found between the modal estimates (natural frequencies and mode shapes) obtained from FDD and SSI techniques;

3. The dynamic behaviour of the tower seems to be significantly affected by the amplitude of excitation. Peculiarly, the natural frequencies identified under the excitation provided by the swinging of bells are slightly lower than the ones identified when the excitation was only provided by wind and microtremors;

4. The comparison between measured and predicted modal parameters was used to verify the assumptions adopted in formulating a F.E. model of the structure. Although this part of the study cannot be considered concluded due to excessive lack of information on the mechanical characteristics of materials, the correlation between theoretical and experimental modal parameters provides fairly accurate and robust verification of the model main assumptions. Hence, the model is a good candidate for use in successive investigation including further non-destructive tests on the materials, more refined sensitivity analysis and the application of structural identification techniques to better assess the structural condition of the tower;

5. The orthotropic elasticity assumption seems more suitable when modelling stone masonry structures. 


\section{Acknowledgements}

The research was supported by the M.I.U.R. (Italian Ministry of University and Research), under grant PRIN06. Furthermore, the authors would like to thank M. Antico, M. Cucchi (Laboratory of Vibrations and Dynamic Monitoring of Structures, Dept. of Structural Engineering, Politecnico di Milano) and Dr. Arch. L. Cantini for their assistance during the field tests. The authors are indebted with Prof. L. Binda for having allowed and encouraged the research.

\section{References}

[1] Binda, L., Saisi, A. \& Tiraboschi, C., Investigation procedures for the diagnosis of historic masonries, Construction and Building Materials, 14(4), pp. 199-233, 2000.

[2] Bendat, J.S. \& Piersol, A.G. Engineering applications of correlation and spectral analysis, Wiley Interscience, $2^{\text {nd }}$ Ed., 1993.

[3] Brincker R., Zhang L.M. \& Andersen P., Modal identification from ambient responses using Frequency Domain Decomposition, Proc. $18^{\text {th }}$ Int. Modal Analysis Conference (IMAC-XVII), pp. 625-630, 2000.

[4] Van Overschee, P. \& De Moor, B., Subspace identification for linear systems: Theory, implementation, applications, Kluwer, 1996.

[5] Ramos, L., Damage identification on masonry structures based on vibration signatures, Ph.D. Thesis, University of Minho, 2007.

[6] Cantieni, R., Experimental methods used in system identification of civil engineering structures, Proc. $1^{\text {st }}$ Int. Operational Modal Analysis Conf. (IOMAC’05), pp. 249-260, 2005.

[7] ARTeMIS Program Overview, http://www.svibs.com, 2006.

[8] Peeters, B., System identification and damage detection in civil engineering structures. Ph.D. Thesis, Katholieke Universiteit Leuven, 2000.

[9] Allemang, R.J. \& Brown, D.L., Correlation coefficient for modal vector analysis, Proc. $1^{\text {st }}$ Int. Modal Analysis Conf. (IMAC-I), pp. 110-116, 1983.

[10] Gentile, C. \& Saisi, A., Ambient vibration testing of historic masonry towers for structural identification and damage assessment, Construction and Building Materials, 21(6), pp. 1311-1321, 2007. 